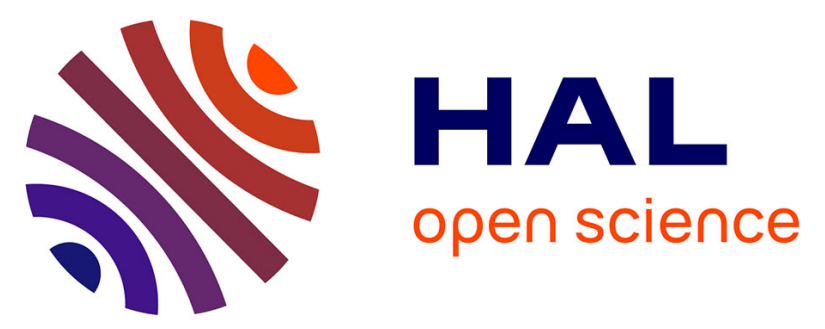

\title{
Effect of porosity on the thermal expansion coefficient: A discussion on the paper 'Effects of mineral admixtures on the thermal expansion properties of hardened cement paste' by Z.H. Shui, R. Zhang, W. Chen, D. Xuan, Constr. Build. Mater. 24(9) (2010) 1761-1767
}

Siavash Ghabezloo

\section{To cite this version:}

Siavash Ghabezloo. Effect of porosity on the thermal expansion coefficient: A discussion on the paper 'Effects of mineral admixtures on the thermal expansion properties of hardened cement paste' by Z.H. Shui, R. Zhang, W. Chen, D. Xuan, Constr. Build. Mater. 24(9) (2010) 1761-1767. Construction and Building Materials, 2010, 24 (9), pp.1796-1798. 10.1016/j.conbuildmat.2010.03.006 . hal-00495092

\author{
HAL Id: hal-00495092 \\ https://hal.science/hal-00495092
}

Submitted on 25 Jun 2010

HAL is a multi-disciplinary open access archive for the deposit and dissemination of scientific research documents, whether they are published or not. The documents may come from teaching and research institutions in France or abroad, or from public or private research centers.
L'archive ouverte pluridisciplinaire HAL, est destinée au dépôt et à la diffusion de documents scientifiques de niveau recherche, publiés ou non, émanant des établissements d'enseignement et de recherche français ou étrangers, des laboratoires publics ou privés. 
Effect of porosity on the thermal expansion coefficient: A discussion on the paper 'Effects of mineral admixtures on the thermal expansion properties of hardened cement paste’ by Z.H. Shui, R. Zhang, W. Chen, D. Xuan, Constr. Build. Mater. 24(9) (2010) 1761-1767

\section{Siavash Ghabezloo*}

BRGM, Natural Risks and $\mathrm{CO}_{2}$ Storage Safety division, Orléans, France

\section{Abstract}

A discussion is presented on the effect of porosity change on the thermal expansion coefficient of porous materials. It is shown that both drained and undrained thermal expansion coefficients normally increase with porosity increase. Consequently the reduction of the thermal expansion coefficient with the porosity increase, as obtained in the experimental results of Shui et al. (Constr. Build. Mater. (2010), 24(9), 17611767), can not be considered as a general trend for the behaviour of porous materials.

Keywords: porosity, thermal expansion coefficient, porous material

\section{Please cite this paper as:}

Ghabezloo S. (2010) Effect of porosity on the thermal expansion coefficient: A discussion on the paper 'Effects of mineral admixtures on the thermal expansion properties of hardened cement paste' by Z.H. Shui, R. Zhang, W. Chen, D. Xuan, Constr. Build. Mater. 24(9) (2010) 1761-1767, Constr. Build. Mater. 24(9), 1796-1798

\footnotetext{
* Siavash Ghabezloo, BRGM-RNSC, 3 avenue Claude Guillemin, BP 36009, F-45060 Orléans Cedex 2, France. Email: s.ghabezloo@brgm.fr
} 


\section{Introduction}

This discussion is presented on the arguments made by Shui et al. [1] on the effect of porosity on the coefficient of thermal expansion of porous materials. In the introduction of their paper, Shui et al. [1] argue that "The CTE of a compacted material is greater than that of a porous material". Moreover in the discussion of the experimental results in section 3.2 of [1], these authors mention that “... more porous materials have lower CTE, because the void could accommodate the internal thermal expansion of material”. For both mentioned arguments a paper published by our group, Ghabezloo et al.[2], is cited. But the mentioned argument about the effect of porosity on the thermal expansion coefficient is not given in our paper and it is not clear how the authors could obtain it from the presented results. Shui et al. [1] present the reduction of the thermal expansion coefficient with the porosity increase, which is obtained from their experimental results, as a general rule for the behaviour of porous materials. However it seems that the generality of this statement is questionable and the experimental conditions in which the measured thermal expansion coefficients decrease with porosity increase should be clearly defined. The aim of this discussion is to clarify the effect of porosity change on the coefficient of thermal expansion of porous materials.

\section{Effect of porosity on thermal expansion coefficient}

When studying the coefficient of thermal expansion of a porous material, the pore fluid drainage conditions should be clearly defined. Two extreme conditions, called drained and undrained conditions can be considered. The drained condition is defined as a condition in which the pore pressure is constant in the material. This condition corresponds to the behaviour of dry porous materials or the case of slow heating of a porous material in a way that the excess pore pressure can be entirely dissipated. The coefficient of drained volumetric thermal expansion $\alpha_{d}$ is defined as:

$$
\alpha_{d}=\frac{1}{V_{0}}\left(\frac{\partial V}{\partial T}\right)_{p_{f}, \sigma}
$$

where $V_{0}$ is the total volume, $T$ is the temperature, $p_{f}$ is the pore pressure and $\sigma$ is the mean stress (positive in compression). Equation (1) corresponds to a drained heating test in which the pore pressure is controlled to remain constant in the sample while the temperature is changes. The variations of the total volume of the sample $V$ with respect to the applied temperature change give the volumetric drained thermal expansion coefficient $\alpha_{d}$. The undrained condition is defined as a condition in which the mass of the fluid phase is constant $\left(d m_{f}=0\right)$. In this condition the discrepancy between the thermal expansion coefficient of the pore fluid and the one of the pore volume generates an excess pore pressure and increases the thermal expansion. The undrained condition corresponds to quick heating of low permeability porous 
materials when the excess pore pressure can not be dissipated. The undrained volumetric thermal expansion coefficient $\alpha_{u}$ is defined as:

$$
\alpha_{u}=\frac{1}{V_{0}}\left(\frac{\partial V}{\partial T}\right)_{m_{f}, \sigma}
$$

The undrained thermal expansion coefficient can be expressed as a function of the other thermo-poroelastic parameters [3][2]:

$$
\alpha_{u}=\alpha_{d}+\phi_{0} B\left(\alpha_{f}-\alpha_{\phi}\right)
$$

where $\phi_{0}$ is the porosity, $B$ is the Skempton coefficient, $\alpha_{f}$ is the pore fluid thermal expansion coefficient and $\alpha_{\phi}$ is the pore volume thermal expansion coefficient [2].

$$
\alpha_{\phi}=\frac{1}{V_{\phi 0}}\left(\frac{\partial V_{\phi}}{\partial T}\right)_{p_{f}, \sigma}
$$

where $V_{\phi}=\phi V$ is the pore volume. In the case of an ideal porous material constituted by one homogeneous solid phase we have $\alpha_{\phi}=\alpha_{d}=\alpha_{s}$, where $\alpha_{s}$ is the thermal expansion coefficient of the solid material [2][3]. The difference between the expansion coefficients $\alpha_{d}$ and $\alpha_{s}$ reflects the difference between the thermal response of the bulk porous medium and that of the solid phase alone [3]. The experimental evaluation of the coefficient $\alpha_{\phi}$ for the general case of a micro-heterogeneous porous material is very difficult [2].

The experimental evaluation of thermal expansion coefficient should be performed under controlled drainage conditions to measure either the drained or the undrained thermal expansion coefficient [2][7]. However in many cases these conditions are not controlled and consequently the measured coefficient is some value between the drained and the undrained thermal expansion coefficient of the tested material. The measured coefficient depends particularly on the permeability of the tested material, sample size and drainage length and rate of heating. The condition is closer to the drained condition for higher permeability, shorter drainage length and slower heating.

The effect of porosity variations on the drained thermal expansion coefficient can be studied first for a simple case of a homogeneous and isotropic porous material. Consider two hollow spheres with the same external radius and different internal radius. The coefficients of thermal expansion of these spheres, which are equivalent to two ideal porous materials with different porosity, can be evaluated by measuring the volume change of these spheres when subjected to a homogeneous temperature change. In the framework of thermoelasticity it can be easily shown that the variation of the total volume of these spheres is independent of the internal radius and is equal to the one of a solid sphere. So the thermal expansion coefficient of an ideal porous material which is made up of one single solid phase is not varied by porosity variations, and is equal to the thermal expansion coefficient of the solid phase. 
The hardened cement paste is a very complex porous material which is constituted by several solid phases [6]. Accordingly it is convenient to study the effect of porosity change on the thermal expansion of more complex porous materials with a heterogeneous microstructure. A literature survey shows that there are very few experimental results on the dependence of the drained thermal expansion to compaction or porosity changes. The experimental results of Wong and Brace [4] show the reduction of the thermal expansion coefficients of very low porosity rocks with confining pressure increase. Considering the limited number of experimental results in the literature, the dependence of thermal expansion on the porosity can be evaluated indirectly by using the temperature dependency of drained compressibility for which more experimental results are available.

The variation of the drained thermal expansion with porosity is related to the variations of this parameter with the stress state using the following relation [2][6]:

$$
\frac{\partial \alpha_{d}}{\partial \phi}=\frac{\partial \alpha_{d}}{\partial \sigma} \cdot \frac{\partial \sigma}{\partial \phi}=-\left[\left(1-\phi_{0}\right) c_{d}-c_{s}\right]^{-1} \cdot \frac{\partial \alpha_{d}}{\partial \sigma}
$$

where $c_{d}$ and $c_{s}$ are respectively the drained compressibility and the unjacketed compressibility. The drained compressibility $c_{d}$ (inverse of the drained bulk modulus $K_{d}$ ) is defined as:

$$
c_{d}=\frac{1}{K_{d}}=-\frac{1}{V_{0}}\left(\frac{\partial V}{\partial \sigma}\right)_{p_{f}, T}
$$

Note that in addition to the mean stress, the porosity can also be varied as a function of the pore pressure or the temperature. However as the objective is to demonstrate the effect of porosity variations on the thermal expansion coefficient, only one of the mechanisms of porosity variation is taken into account. Consequently in equation (5) the variations of porosity with the temperature and the pore pressure are ignored for the sake of simplicity, but the objective of the present discussion is not influenced by this simplification.

The variations of the drained thermal expansion with the mean stress in equation (5) can be related to the variations of the drained compressibility with temperature. Using equations (1) and (6) the following relation can be obtained:

$$
\frac{\partial \alpha_{d}}{\partial \sigma}=-\frac{\partial c_{d}}{\partial T}
$$

Inserting equation (7) in equation (5) the following expression is obtained:

$$
\frac{\partial \alpha_{d}}{\partial \phi}=\left[\left(1-\phi_{0}\right) c_{d}-c_{s}\right]^{-1} \cdot \frac{\partial c_{d}}{\partial T}
$$

Considering the Voigt upper bound [5] we have $\left(1-\phi_{0}\right) c_{d}-c_{s} \geq 0$. As an example, for the poroelastic properties of the hardened cement paste studied by Ghabezloo et al. [6], we obtain $\left[\left(1-\phi_{0}\right) c_{d}-c_{s}\right]^{-1}=26.7 \mathrm{GPa}$. Consequently from equation (8) the variations of drained thermal expansion with the porosity follow the same trend as the variations of the drained compressibility with temperature. The existing experimental results in many cases show the reduction of the elastic modulus (increase of the 
drained compressibility) with temperature increase, i.e. $\partial c_{d} / \partial T>0$. For the case of the cement-based materials, we can for instance mention the experimental results of Odelson et al. [8] and Savva et al. [9] which show the decrease of the elastic modulus (i.e. increase of the compressibility) with temperature increase. From equation (8) we find $\partial \alpha_{d} / \partial \phi>0$, i.e., the drained thermal expansion coefficient increases with porosity. This is compatible with a part of the experimental results of Shui et al. [1], presented in their Figure 4.3-c.

The effect of the porosity on the undrained thermal expansion coefficient $\alpha_{u}$ can also be studied. The thermal expansion coefficient of water is about one order of magnitude greater than the one of the most solids. This discrepancy resulted in a phenomenon of thermal pressurization, and consequently a greater thermal expansion, when the saturated porous material is subjected to heating in undrained condition. Consequently, the undrained thermal expansion coefficient is greater than the drained coefficient and is directly related to the quantity of the fluid in the pore volume of the material, as can be seen in equation (3). From this equation one can see that the variation of the undrained thermal expansion coefficient with the porosity $\partial \alpha_{u} / \partial \phi$ depends on the variations of the drained thermal expansion $\alpha_{d}$, the pore volume thermal expansion $\alpha_{\phi}$ and the Skempton coefficient $B$ with the porosity.

$$
\frac{\partial \alpha_{u}}{\partial \phi}=\frac{\partial \alpha_{d}}{\partial \phi}+\left(B+\phi_{0} \frac{\partial B}{\partial \phi}\right)\left(\alpha_{f}-\alpha_{\phi}\right)-\phi_{0} B \frac{\partial \alpha_{\phi}}{\partial \phi}
$$

It has been already shown that in most cases $\partial \alpha_{d} / \partial \phi>0$. The Skempton coefficient is always positive, $B>0$ and generally increases with porosity increase, $\partial B / \partial \phi>0$. The increase of Skempton coefficient with porosity is due to the increase of the compressibility of the material with porosity increase. The fluid thermal expansion coefficient $\alpha_{f}$ is significantly greater than the pore volume thermal expansion coefficient $\alpha_{\phi}$, i.e., $\alpha_{f}-\alpha_{\phi}>0$. The discrepancy between the thermal expansions $\alpha_{f}$ and $\alpha_{\phi}$ is more significant for the hardened cement paste for which the pore fluid thermal expansion coefficient is anomalously higher than the one of the bulk fluid [2][10]. This anomalous thermal expansion behaviour is attributed to the confinement of a part of the pore fluid in very small pores $(<15 \mathrm{~nm})$. The trend of variation of the pore fluid thermal expansion with the porosity $\partial \alpha_{\phi} / \partial \phi$ is unknown. However, the significant discrepancy between $\alpha_{f}$ and $\alpha_{\phi}$ makes the second part of equation (9) a dominant term and consequently the undrained thermal expansion coefficient in most cases should have an increasing trend with porosity increase, i.e. $\partial \alpha_{u} / \partial \phi>0$. For the case of a simple porous material for which $\alpha_{\phi}=\alpha_{d}$, equation (9) simplifies to the following equation:

$$
\frac{\partial \alpha_{u}}{\partial \phi}=\frac{\partial \alpha_{d}}{\partial \phi}\left(1-\phi_{0} B\right)+\left(B+\phi_{0} \frac{\partial B}{\partial \phi}\right)\left(\alpha_{f}-\alpha_{d}\right)
$$

Knowing that $\phi_{0} B<1$, equation (10) results in a definitive increase of the undrained thermal expansion coefficient with the porosity. 
We have seen that in both drained and undrained conditions, the thermal expansion coefficients of a porous material normally increase with porosity. However there are some experimental artefacts which may result in the measurement of thermal expansion coefficients that decrease with porosity increase. As mentioned before, when the drainage conditions of the test are not controlled, the measured thermal expansion coefficient is some value between the drained and the undrained coefficient. From equation (3) we can see that the undrained thermal expansion coefficient is greater that the drained coefficient. Consequently, for the same material one can measure the thermal expansion coefficients that increase with the heating rate and the drainage length. Now consider two porous materials with similar solid phases and different porosities. Considering that the permeability increases with the porosity, the drainage condition for the material with lower porosity is closer to the undrained condition. Consequently the measured thermal expansion coefficient for this material is higher that the one measured for the one with higher porosity. This experimental artefact results in erroneous measurement of thermal expansion coefficients that decrease with porosity increase.

\section{Conclusions}

The effect of porosity change on the thermal expansion coefficient of porous materials is discussed. It has been demonstrated that both drained and undrained thermal expansion coefficients normally increase with the porosity. However, some evolution processes that change the microstructure and the constituents of a porous material, e.g. chemical reactions, may result is different evolutions of the thermal expansion coefficient with the porosity. Moreover, when the test drainage conditions are not controlled, some experimental artefacts may result in erroneous measurement of thermal expansion coefficients that decrease with porosity increase. Consequently, the decreasing trend of the evaluated thermal expansion coefficient with the porosity, as presented by Shui et al. [1], is limited to the studied material and the performed experimental study and can not be considered as a general property for porous materials.

\section{References}

[1] Z.H. Shui, R. Zhang, W. Chen, D. Xuan, Effects of mineral admixtures on the thermal expansion properties of hardened cement paste. Constr. Build. Mater. 2010, doi:10.1016/j.conbuildmat.2010.02.012

[2] S. Ghabezloo, J. Sulem, J. Saint-Marc. The effect of undrained heating on a fluid-saturated hardened cement paste. Cement and Concrete Research 2009;39:54-64.

[3] D.F. McTigue, Thermoelastic response of fluid-saturated porous rock. Journal of Geophysical Research 1986;91(B9):9533-9542.

[4] T.F. Wong, W.F. Brace, Thermal expansion of rocks: some measurements at high pressure, Tectonophysics, 1979;57:95-117.

[5] A. Zaoui, Continuum Micromechanics: Survey, Journal of Engineering Mechanics 2002; 128(8):808-816. 
[6] S. Ghabezloo, J. Sulem, S. Guedon, F. Martineau, J. Saint-Marc, Poromechanical behaviour of hardened cement paste under isotropic loading. Cement and Concrete Research 2008;38:1424-1437.

[7] S. Ghabezloo, J. Sulem, Stress dependent thermal pressurization of a fluid-saturated rock, Rock Mechanics and Rock Engineering 2009;42:1-24

[8] J.B. Odelson, E.A. Kerr, W. Vichit-Vadakan, Young's modulus of cement paste at elevated temperatures, Cement and Concrete Research 2007;37:258-263.

[9] A. Savva, P. Manita, K.K. Sideris, Influence of elevated temperatures on the mechanical properties of blended cement concretes prepared with limestone and siliceous aggregates, Cement \& Concrete Composites 2005;27:239-248.

[10] J.J. Valenza II, G.W. Scherer, Evidence of anomalous thermal expansion of water in cement paste, Cement and Concrete Research 2005;35:57-66. 